\title{
Research on The Drive System of Piezoelectric Injector
}

\author{
Shang $\mathrm{Hao}^{1}$, Ying Huang ${ }^{2}$, and Shuang-dong Zhang ${ }^{3^{*}}$ \\ ${ }^{1}$ School of Information Engineering, Wuhan University of Technology, Key Laboratory of Fiber Optic \\ Sensing Technology and Information Processing (Wuhan University of Technology), Ministry of \\ Education Wuhan ,China, 430070 \\ ${ }^{2}$ School of Information Engineering, Wuhan University of Technology, Wuhan, China, 430070 \\ ${ }^{3}$ School of Information Engineering, Wuhan University of Technology, Wuhan, China, 430070 \\ ${ }^{*}$ Corresponding author
}

Keywords: Piezoelectric Actuator; Injection; Driving Mode; Control Strategy

\begin{abstract}
The paper analyzes two kinds of driving mode of piezoelectric injectors ccording to the characteristics of the piezoelectric actuator, then compares the advantages and disadvantages of the fixed duty cycle PWM switching type, variable duty cycle PWM switching and driver-based adaptive current PWM switching type, chooses voltage and current double closed-loop control strategy. The hardware and software design of high pressure common rail piezo injector driving control system were completed, as well as the experiments of different driving implementation were finished based on the designed driving control system. The results show that driver-based adaptive current PWM switching type is able to control the piezoelectric actuator deformation and the strain rate flexibly, ensure the energy distribution in the charge-discharge process of piezoelectric actuator gets more balanced.
\end{abstract}

\section{Introduction}

With the energy crisis even more serious, since the diesel engine has combustion efficiency and therefore the application ranges more widely. In order to solve the problem of vibration and noise of the diesel engine after the injection pressure increase, the multi-injection is widely applied to the direct injection diesel engine system. The research under the context of Euro-IV and higher emission regulations shows that: The consistency and the precise control of fuel injection contribute to the reduction of nitrogen oxides (NOx) emissions. Compared with the most reliable high-speed electromagnetic injector, Piezoelectric injectors can reduce diesel emissions by $20 \%$ of harmful substances in the 16OMPa system pressure and its flexible adjustment can accurately control the quantity of fuel injection, on heavy-duty vehicles can achieve Euro-IV emission standards without the use of post-processing equipment, while improving $5 \%$ power, reducing $3 \mathrm{Db}(\mathrm{A})$ noise and saving $3 \%$ fuel consumption. [1-3]

\section{Driving Circuit Principle}

The main control component of the high pressure common rail piezoelectric injector is the piezoelectric actuator, which is made by several hundred pieces of piezoelectric ceramics in parallel [4], so piezoelectric actuators also have positive pressure electrical effect and inverse piezoelectric effect. Positive piezoelectric effect is the piezoelectric ceramic will have the same positive and negative charge in both ends when the external force is applied to the piezoelectric ceramic, and the amount of charge is proportional to the size of the external force. [5] The inverse piezoelectric effect refers to the piezoelectric ceramic is the piezoelectric ceramics will produce deformation when placed in the electric field, the deformation and electric field size is almost proportional.

The piezoelectric actuator driving system at work requires precise control in the injection process of piezoelectric actuators, mainly on the driving circuit voltage and current control. The driving 
circuit designed according to PWM switching principle and the analysis of driving method schematic diagram shown in Figure.1.

As can be seen from Figure.1, The entire driving circuit mainly includes BOOST module, actuator charge and discharge module, ECU master module. From the circuit characteristics point of view, piezoelectric actuators are capacitive loads, so the drive of the piezoelectric actuators is the process of its charge and discharge, ECU module control charge and discharge process works as follows:

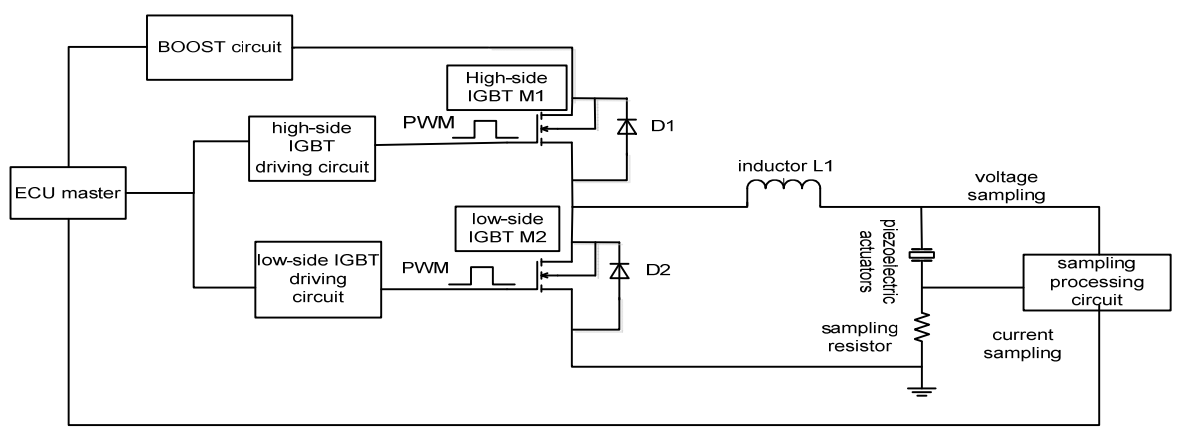

Figure.1 Principle diagram of driving circuit

During charging, the PWM signal from the master chip first turns on the high-side IGBT transistor M1, while the low-side IGBT transistor M2 is turned off, the high voltage generated by the BOOST circuit is applied to the piezoelectric actuator through the inductor L1. When M1 is closed, L1 generates induced electromotive force to form a loop through the piezoelectric actuator and diode D2 to continue to charge the piezoelectric actuator.

During discharging, The PWM signal from the master chip turns off M1 first, and then turnsonM2, the energy in the piezoelectric actuator is transferred to L1. L1 charges the high voltage capacitance through diode D1 to achieve energy recovery when M2 is off. [6]

Piezoelectric actuator current driving mode using multiple PWM control signal driven implementation. Multiple PWM driving mode (Multi-peak current driving mode) divides one single PWM control signal into a plurality of control cycles to perform a small charge and discharge process once per control cycle. This method is suitable for pre-injection, main injection and post-injection of different spray process, which has more flexible control methods to limit the size of the driving current, extend the life of piezoelectric actuators. Multi-peak current driving mode can also achieve the uniform distribution of energy during charge and discharge process, so that the whole system has high reliability and energy efficiency. Therefore, this paper chooses the multi-peak current driving method.

As can be seen in Figure.2, multi-peak current drive mode can make the charge and discharge current in each cycle more balanced. Due to the piezoelectric actuator at both ends of the pressure difference is large which resulting in the initial state of the current over shoot in the first charge and discharge cycle, so that the piezoelectric actuator premature aging and we set a smaller duty value in the experiment. The voltage difference between the two ends of the piezoelectric actuator becomes smaller with the progress of charge and discharge corresponding to set a larger value of duty cycle to ensure that the piezoelectric actuator charge and discharge speed. Multi-peak current driving only needs to control the number and size of the duty cycle of two parameters but vulnerable to changes in the system. As the repeated injection, the piezoelectric material will produce thermal effects resulting in fuel injection system instability, so the system needs to adjust the parameters of adaptive control strategy. 


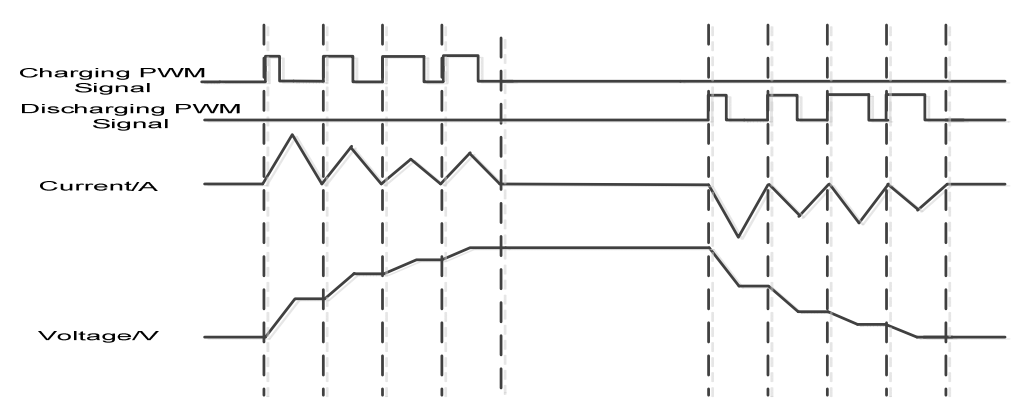

Figure. 2 voltage and current waveform in multi-peak current driving mode

\section{Parameter Self-adaptive Control Strategy}

The main control component of the high pressure common rail piezoelectric injector is the piezoelectric actuator, which is made by several hundred pieces of piezoelectric ceramics in parallel, so piezoelectric actuators also have positive pressure electrical effect and inverse piezoelectric effect. Positive piezoelectric effect is the piezoelectric ceramic will have the same positive and negative charge in both ends when the external force is applied to the piezoelectric ceramic, and the amount of charge is proportional to the size of the external force. The inverse piezoelectric effect refers to the piezoelectric ceramic is the piezoelectric ceramics will produce deformation when placed in the electric field, the deformation and electric field size is almost proportional.

The parameter self-adaptive control strategy includes off-line calibration and on-line adjustment of PWM parameters. In the offline phase, the system defaults are calculated and analyzed, and then the parameters are calibrated by the following steps:

Step 1: the first cycle as the current control cycle;

Step 2: ECU outputs the current cycle control signal according to preset parameters and samples the voltage and current across the piezoelectric material;

Step 3: Compare the sampled value with the preset value of the system. If it does not meet the requirement, adjust the parameters adaptively;

Step 4: Go to step 2, until the sampled value meets the system default size;

Step 5: Save the adjustment result while use the next cycle as the current control cycle

and repeat step 2 until all pulse cycle control parameters have been adjusted;

Step 6: If the sampling voltage is still lower than the required voltage range after the last control cycle increase the number of pulses.

The ECU can drive the piezoelectric actuators according to the calibration results after the offline phase is completed. But the piezoelectric actuator due to aging or vehicle engine temperature rise caused by their own characteristics change in the actual operation, so the process requires parameters online adjustment process. The injection parameters are adjusted only between the two injection cycles in order to ensure the even inertia of the fuel injection during the vehicle running during the online adjustment of the control parameters. The current injection process in the drive current sampling value stored before the start of the next fuel injection, ECU feedback value based on the last fuel injection cycle current, the next injection cycle control parameters to be amended through repeated adjustments, The injection process is controlled within the normal range.

\section{System Experiment}

Figure. 3 for the system physical map, the design of the software is completed by using a fixed duty cycle current driving mode based on the hardware design of the system, which takes a variable duty cycle flow driving mode, an adaptive parameter variable duty cycle and multiple peak current driving mode to drive the piezoelectric actuators to verify the theoretical analysis according to the piezoelectric actuator voltage and current size. The pulse frequency of the PWM is $50 \mathrm{KHz}$ and there are 6 pulses in the charge-discharge process of the piezoelectric actuator in the experiments, the driving voltage and the 
current in adaptive parameter variable duty cycle and multiple peak current driving mode is shown in Figure.4.

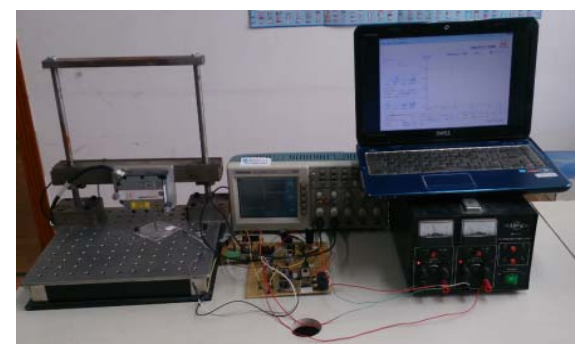

Figure. 3 system physical diagram

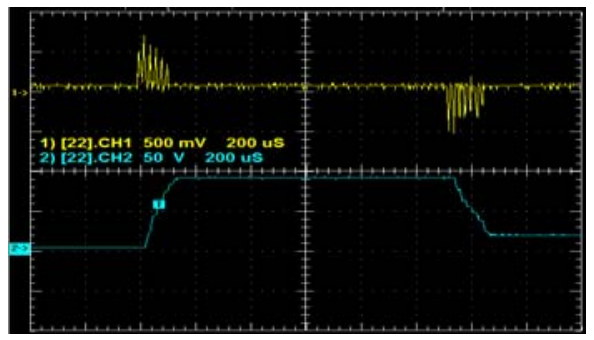

Figure.4 voltage and current waveform

Figure. 4 shows the waveform after the parameter adjustment. It can be seen that the driving voltage changes step-by-step; the charging and discharging time is about 120us; the current is more stable and within the target range during the charging and discharging cycle.

In this paper, Iridium ILD2300-2 laser displacement sensor is used to detect the displacement of piezoelectric actuators during charging and discharging. As shown in Figure.5, the maximum stroke is 6um and the response time of the piezoelectric actuator is about 120us when the voltage across the piezoelectric actuator reaches $100 \mathrm{~V}$; the injection time is $1050 \mathrm{us}$ and the driving current is not needed in the injection process; the injection end time is about 105us, in line with the piezoelectric injector response to the requirements of the actuator but power consumption is relatively low.

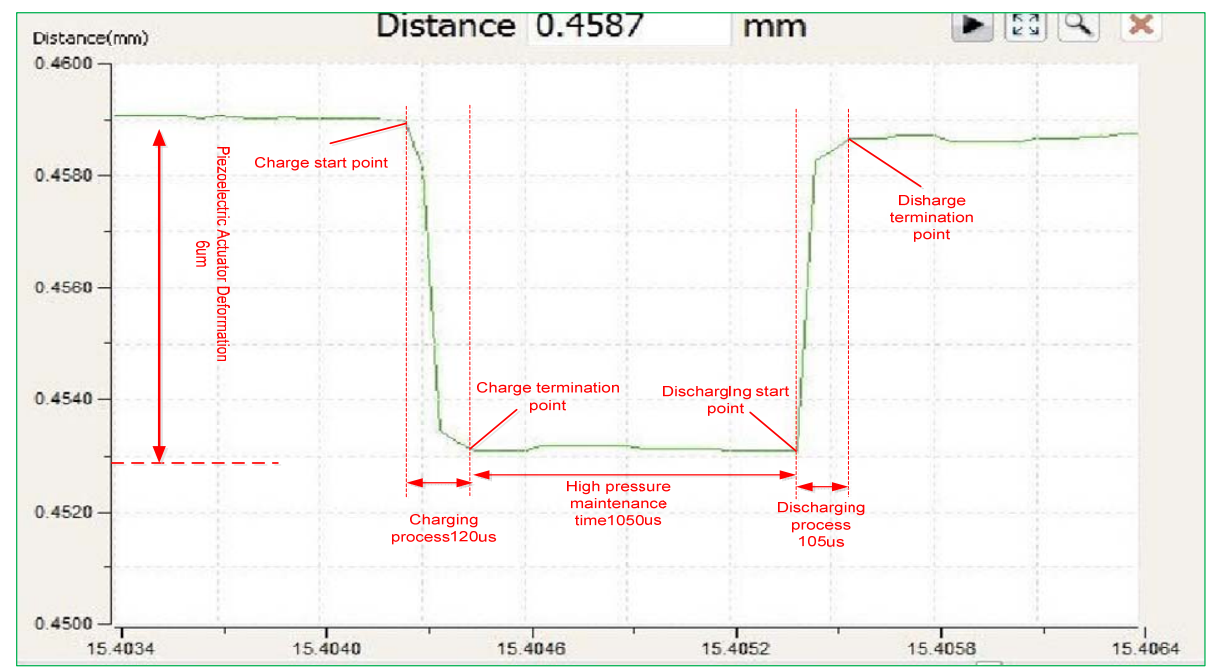

Figure.5 Stroke change of piezoelectric actuator

\section{Conclusion}

The conclusion shows that the flexible control of the PWM pulse can better control the drive current size through the analysis of the piezoelectric injection system, while the piezoelectric actuator should be limited to 120usto meet the needs of rapid fuel injection; The parameters of adaptive adjustment strategy for each charge and discharge cycle monitoring, the drive current distribution more evenly to improve the reliability of the entire drive system; Piezoelectric actuators do not need to drive the current to maintain fuel injection open, which can effectively reduce power consumption and save energy. 


\section{References}

[1] Tan Xu-Guang Sang Hai-Lang, QIU Tao, Fan Zhi-Qiang, Yin Wen-Hui. The Impact of Common Rail System's Control Parameters on the Performance of High-power Diesel [J]. Energy Procedia, 2012, 16:.

[2] Plamondon E, Seers P. Development of a simplified dynamic model for a piezoelectric injector using multiple injection strategies with biodiesel/diesel-fuel blends [J]. Applied Energy, 2014, 131(9):411-424.

[3] A. Ferrari, A. Mittica, E. Spessa. Benefits of hydraulic layout over driving system in piezo-injectors and proposal of a new-concept CR injector with an integrated Minirail[J]. Applied Energy, 2013, 103:.

[4] A. Ferrari, A. Mittica. FEM modeling of the piezoelectric driving system in the design of direct-acting diesel injectors [J]. Applied Energy, 2012, 99:.

[5] P. K. Panda, B. Sahoo. PZT to Lead Free Piezo Ceramics: A Review [J]. Ferroelectrics, 2015, 4741:.

[6] O. Armas, S.Martinez, C. Mata. Effect of an ethanol-biodiesel-diesel blend on a common rail injection system [J]. Fuel Processing Technology, 2011, 9211:. 